\title{
Uso de biorregulador e seus reflexos na produção e na qualidade de sementes de trigo
}

Use of bioregulator and its reflections on the production and quality of wheat seeds

\author{
S. de Oliveira*; E. S. Lemes; E. H. das Neves; R. Ritter; A. O. de Mendonça; G. E. \\ Meneghello \\ Universidade Federal de Pelotas/Programa de Pós-graduação Ciência e Tecnologia de Sementes, Capão do Leão-Rio \\ Grande do Sul, Brasil \\ *lemes.elisa@yahoo.com.br
}

(Recebido em 18 de julho de 2019; aceito em 27 de janeiro de 2020)

\begin{abstract}
A região sul do Rio Grande do Sul não possui tradição na produção de trigo, e em função do aumento na área de produção da soja, tem-se aumentado o cultivo de trigo nestes locais. Neste contexto, procura-se cada vez mais aprimorar as técnicas de produção nessas áreas, buscando alternativas para que se atinja o máximo de produção com o mínimo de impacto possível. Diante disso, objetivou-se explorar os efeitos da aplicação do biorregulador Stimulate ${ }^{\circledR}$, via tratamento de sementes e aplicações foliares, na produtividade e na qualidade das sementes de trigo produzidas em solos de várzea. $\mathrm{O}$ delineamento experimental utilizado foi inteiramente casualizado em esquema fatorial, sendo o fator A constituído pelas cultivares de trigo e o fator B pelas doses de biorregulador via tratamento de sementes ou foliar. Os experimentos foram avaliados quanto aos componentes do rendimento e a qualidade fisiológica das sementes produzidas. A utilização de Stimulate ${ }^{\circledR}$ exerce efeito diferenciado nas cultivares de trigo, influenciado pela genética. O biorregulador Stimulate ${ }^{\circledR}$ aplicado via foliar nos estádios V3 e R1, nas doses próximas de $450 \mathrm{~mL} \mathrm{ha}^{-1}$, ou via tratamento de sementes até $1000 \mathrm{~mL} 100 \mathrm{~kg}$ sementes ${ }^{-1}$, podem promover acréscimos no rendimento de sementes, no peso de mil sementes e na qualidade fisiológica das sementes produzidas, dependendo da cultivar de trigo.

Palavras-chave: tratamento de sementes, aplicação foliar, regulador de crescimento.
\end{abstract}

The southern region of Rio Grande do Sul is not a traditional region of wheat production, and due to the increase in the area of soybean production, wheat cultivation has increased in these areas. In this context, it is increasingly sought to improve production techniques in these areas, seeking alternatives to achieve maximum production with the least possible impact. The aim of this study was to evaluate the effects of the application of the Stimulate ${ }^{\circledR}$ bioregulator, through seed coating and leaf applying, on the productivity and quality of the wheat seeds produced in lowland soils. The experimental model used was a completely randomized in a factorial scheme, being the factor A constituted by the wheat cultivars and the factor B by the bioregulator doses, through seed coating or leaf applying. Yield components and the physiological quality of the produced seeds were analyzed. The use of Stimulate ${ }^{\circledR}$ has a different effect on wheat cultivars, influenced by its genetics. Stimulate ${ }^{\circledR}$ bioregulator through leaf applying at stages V3 and R1, at doses around of $450 \mathrm{~mL} \mathrm{ha}^{-1}$, or seed coating up to $1000 \mathrm{~mL} 100 \mathrm{~kg} \mathrm{seed}^{-1}$, can promote increases in seed yield, thousand seed weight and on the physiological quality of the seeds produced, depending on the wheat cultivar. Keywords: seed coating, leaf applying, plant growth regulator.

\section{INTRODUÇÃO}

O trigo (Triticum aestivum L.) é um dos principais produtos utilizados na alimentação humana e animal, sendo a farinha, um dos seus subprodutos, a principal matéria-prima para elaboração de alimentos, na forma de pães, biscoitos, bolos e massas, alimentos que fazem parte da base da pirâmide alimentar [1]. No Brasil, essa cultura contribui significativamente na economia do país, por ser um dos principais cereais de inverno. No entanto, a produtividade do trigo varia anualmente e de região para região, em função, principalmente, da deficiência nutricional, doenças, pragas e fertilidade do solo [2].

Devido à grande demanda por alimentos, faz-se necessário expandir e aumentar a produção de trigo para outras regiões que até então não possuem tradição de produção da cultura, diminuindo com isso a dependência externa. Nesse sentido, nos últimos anos as empresas aumentaram as pesquisas, com o intuito de desenvolver materiais que se adaptem a outras condições de clima e ambiente, principalmente para o centro oeste. Um dos principais insumos a ser adquirido pelos 
produtores são as sementes, as quais devem possuir alta qualidade, sendo o tratamento de sementes, uma técnica que contribui para potencializar o desempenho das mesmas. De acordo com Peske et al. (2012) [3] esta técnica é uma realidade, que tem como principal objetivo a proteção não somente das sementes, mas também das plântulas logo após sua emergência. Além disso, o tratamento de sementes possibilita a aplicação conjunta de vários produtos como fungicida, inseticida, micronutrientes, inoculantes e biorreguladores [4].

Além dos tradicionais produtos utilizados no tratamento de sementes e em aplicações foliares como é o caso dos fungicidas, os inseticidas e nutrientes, outros produtos estão sendo utilizados na agricultura para contemplar alguma deficiência ou potencializar os cultivos. Os reguladores de crescimento, também chamados de bioestimulante ou biorreguladores, podem ser aplicados via tratamento de sementes e/ou em aplicações foliares. Os hormônios vegetais produzidos pelas plantas e os reguladores de crescimento e/ou biorreguladores, produzidos sinteticamente, participam de processos do metabolismo vegetal, incluindo a divisão celular, morfogênese, alongamento, biossíntese de compostos e senescência [5, 6]. Estes produtos podem ainda auxiliar na expressão do potencial genético das cultivares, podendo ainda promover alterações nos processos vitais e estruturais, proporcionando um equilíbrio hormonal, estimulando assim o desenvolvimento do sistema radicular [7, 8], o que pode contribuir para o aumento de absorção de água e de nutrientes pelas plantas, dando condições para que as plantas tenham maior resistência para enfrentar possíveis estresses hídricos e efeitos residuais de herbicidas no solo [9].

Vários trabalhos têm destacado a aplicabilidade e uso de biorregulador ou reguladores vegetais em culturas como a do milho, feijão, algodão, soja, arroz, entre outras [8, 10, 11, 12], no entanto, na literatura não se encontram muitos resultados de pesquisa na cultura do trigo na região sul do Rio Grande do Sul, a qual não é uma região com tradição na produção da cultura, e em função do aumento na área de produção da soja, tem-se aumentado o cultivo de trigo nestes locais. Desta forma, o objetivo do trabalho foi avaliar os efeitos da aplicação de biorregulador na cultura do trigo, via tratamento de sementes e aplicações foliares, em cultivo de campo, na qualidade das sementes e na produtividade das plantas.

\section{MATERIAL E MÉTODOS}

Os experimentos foram realizados na safra de inverno de 2014, sendo conduzido em campo, no Centro Agropecuário da Palma, Capão do Leão, RS e os testes de qualidade realizados no Laboratório Didático de Análise de Sementes (LDAS), ambos pertencentes ao Departamento de Fitotecnia da Faculdade de Agronomia Eliseu Maciel (FAEM) da Universidade Federal de Pelotas (UFPel). Foram utilizadas sementes de trigo das cultivares TBIO Itaipu e TBIO Mestre. Previamente ao tratamento de sementes e semeadura foi realizado teste de germinação para verificar a porcentagem inicial de germinação das sementes, sendo que a cultivar TBIO Itaipu apresentava $91 \%$ de germinação, enquanto que a cultivar TBIO Mestre apresentava $92 \%$ de germinação.

Os experimentos foram conduzidos com o uso do biorregulador Stimulate ${ }^{\circledR}$ (Ácido 4-indol-3ilbutírico + ácido giberélico + cinetina), sendo a aplicação realizada via tratamento de sementes e via pulverização foliar.

\subsection{Experimento 1- tratamento de sementes}

$\mathrm{O}$ delineamento experimental utilizado foi o inteiramente casualizado em esquema fatorial $\mathrm{A} x$ B (Fator A - cultivares de trigo: TBIO Itaipu e TBIO Mestre; Fator B - doses de biorregulador, utilizando-se para isso o produto comercial Stimulate ${ }^{\circledR}: 0,250,500,750$ e $1000 \mathrm{~mL} 100 \mathrm{~kg}$ sementes $^{-1}$, com quatro repetições.

As sementes foram tratadas com as doses do biorregulador, seguindo a metodologia sugerida por Nunes (2005) [13] que consiste num método manual de tratamento utilizando sacos plásticos (3L), nos quais foram depositadas as devidas doses de biorregulador, com um volume de calda equivalente a $1000 \mathrm{~mL} 100 \mathrm{~kg}^{-1}$ sementes $^{-1}$, o qual foi completado com água $(1000,750,500,250 \mathrm{e}$ $0 \mathrm{~mL} 100 \mathrm{~kg}$ sementes ${ }^{-1}$, respectivamente). As doses foram aplicadas diretamente no fundo de um saco plástico e espalhados, sendo as sementes acondicionadas diretamente no interior do saco, 
agitando-as por 3 minutos. Posteriormente ao tratamento, os sacos plásticos foram abertos permitindo que as sementes secassem a temperatura ambiente, por um período de 24 horas.

Após o tratamento das sementes, foi realizada análise da qualidade fisiológica inicial das mesmas, através do teste de primeira contagem de germinação (PCG), germinação $(\mathrm{G})$ e vigor, para tanto, foram realizadas as seguintes avaliações: teste de frio (TF), envelhecimento acelerado (EA), comprimento total das plântulas (CTP), comprimento da parte aérea (CPA) e de raiz (CR). As metodologias destes testes serão descritas na sequência.

A semeadura foi realizada mecanicamente com semeadora de parcelas, utilizando uma população de 330.0000 planta ha ${ }^{-1}$ e com adubação de base utilizando $300 \mathrm{~kg} \mathrm{ha}^{-1}$ de NPK, conforme a análise química do solo, sendo as demais adubações nitrogenadas em cobertura realizadas no perfilhamento e 15 dias após. As parcelas experimentais foram constituídas de nove linhas com cinco metros de comprimento, espaçadas em 0,17 m entre si. A área útil de cada parcela constituiu-se das cinco linhas centrais eliminando-se $0,50 \mathrm{~m}$ das extremidades, sendo o restante considerado como bordadura.

Durante todo o ciclo da cultura foi realizado o manejo, de acordo com as recomendações técnicas da cultura, conduzindo o experimento até a fase de maturação de campo, sendo então realizada a colheita das sementes. Para o controle de plantas daninhas, em pós emergência, foi utilizado Iodosulfuron-methyl na dose de $80 \mathrm{~g} \mathrm{ha}^{-1}$ e 2,4-D, na dose de $600 \mathrm{~mL}^{-1}$; controle de doenças através do uso do produto Azoxystrobin + Cyproconazole, sendo realizadas duas aplicações na dose de 300 $\mathrm{mL} \mathrm{ha}{ }^{-1}$, sendo uma no início do florescimento e a outra no enchimento de grãos. O controle de insetos, utilizando o produto Tiametoxam + Lambda-Cialotrina na dose de $150 \mathrm{~mL} \mathrm{ha}^{-1}$.

\subsection{Experimento 2- Pulverização foliar}

$\mathrm{O}$ delineamento experimental utilizado foi o inteiramente casualizado em esquema fatorial $\mathrm{A} x$ $\mathrm{B}$ (Fator A - cultivares de trigo: TBIO Itaipu e TBIO Mestre; Fator B - doses de biorregulador via aplicação foliar: $0 ; 187,5 ; 375 ; 562,5$ e $750 \mathrm{~mL} \mathrm{ha}^{-1}$, com quatro repetições.

A semeadura foi realizada mecanicamente com semeadora de parcelas, utilizando uma população de 330.0000 planta ha $^{-1}$ e com adubação de base utilizando $300 \mathrm{~kg} \mathrm{ha}^{-1}$ de NPK, conforme a análise química do solo, sendo as demais adubações nitrogenadas em cobertura realizadas no perfilhamento e 15 dias após. As parcelas experimentais foram constituídas de nove linhas com cinco metros de comprimento, espaçadas em 0,17 m entre si. A área útil de cada parcela constituiu-se das cinco linhas centrais eliminando-se $0,50 \mathrm{~m}$ das extremidades, sendo o restante considerado como bordadura.

Durante todo o ciclo da cultura foi realizado o manejo, de acordo com as recomendações técnicas da cultura, conduzindo o experimento até a fase de maturação de campo, sendo então realizada a colheita das sementes. Para o controle de plantas daninhas, em pós emergência, foi utilizado Iodosulfuron-methyl na dose de $80 \mathrm{~g} \mathrm{ha}^{-1}$ e 2,4-D, na dose de $600 \mathrm{~mL}^{-1}$; controle de doenças através do uso do produto Azoxystrobin + Cyproconazole, sendo realizadas duas aplicações na dose de 300 $\mathrm{mL} \mathrm{ha}{ }^{-1}$, sendo uma no início do florescimento e a outra no enchimento de grãos. O controle de insetos, utilizando o produto Tiametoxam + Lambda-Cialotrina na dose de $150 \mathrm{~mL} \mathrm{ha}^{-1}$.

\subsection{Variáveis avaliadas}

Em ambos os experimentos, os componentes de rendimento foram avaliados através de contagem direta o número de espigas por planta, sendo estas trilhadas manualmente e então realizada a determinação do peso de sementes por planta. Além destes, foi realizada a avaliação da qualidade das sementes através das seguintes metodologias: Teste de germinação $(G)$ : realizado segundo as Regras para Análise de Sementes - RAS [14]. Primeira contagem da germinação $(P C G)$ : realizado conjuntamente ao teste de germinação. Teste de frio $(T F)$ : conduzido conforme metodologia de Cícero e Vieira (1994) [15]. Envelhecimento acelerado (EA): realizado de acordo com Marcos Filho (2015) [16]. Comprimento de parte área e raiz (CPA e CR) e Emergência em campo (EC): conduzido conforme Nakagawa (1999) [17]. Peso de 1000 sementes: realizado de acordo com o indicado nas Regras para Análise de Sementes - RAS [14]. 


\subsection{Procedimento estatístico}

Os dados de cada experimento foram analisados quanto à normalidade e homocedasticidade e, posteriormente, submetidos à análise de variância $(\mathrm{p}<0,05)$. Sendo significativo foi realizada comparação de médias para os fatores qualitativos através do teste de Tukey e regressão polinomial para os fatores quantitativos, ambos a $5 \%$ de probabilidade. Dados em percentagem oriundos da qualidade fisiológica foram submetidos à transformação arc.sen (raiz x/100).

\section{RESULTADOS E DISCUSSÃO}

\subsection{Experimento 1- tratamento de sementes}

Para a qualidade inicial das sementes tratadas evidenciou-se que não houve significância dos fatores para o teste de frio (dados não apresentados). Para as demais variáveis avaliadas nas sementes tratadas com doses de biorregulador, não se verificou interação entre os fatores estudados. No entanto houve efeito principal de cultivar para as variáveis primeira contagem de germinação, germinação e envelhecimento acelerado, onde a cultivar TBIO Mestre foi superior a TBIO Itaipu (Tabela 1).

Constatou-se interação entre os fatores estudados para o comprimento de parte aérea, onde a cultivar TBIO Mestre foi superior a cultivar TBIO Itaipu em todas as doses aplicadas (Tabela 1). Já para o comprimento de raiz, observa-se efeito principal para o fator cultivar, onde a cultivar TBIO Mestre apresentou maior tamanho, sendo superior a cultivar TBIO Itaipu.

Tabela 1. Qualidade inicial das sementes tratadas através dos testes de primeira contagem de germinação $(P C G)$, germinação $(G)$, teste de frio $(T F)$, envelhecimento acelerado $(E A)$, comprimento da parte aérea (CPA) e comprimento de raiz (CR) de plântulas de trigo das cultivares TBIO Itaipu e TBIO Mestre.

\begin{tabular}{ccccccccccc}
\hline \multirow{2}{*}{ Dose** } & \multicolumn{2}{c}{ PCG $(\%)$} & \multicolumn{2}{c}{$\mathrm{G}(\%)$} & \multicolumn{2}{c}{ EA $(\%)$} & \multicolumn{2}{c}{ CPA $(\mathrm{cm})$} & \multicolumn{2}{c}{ CR $(\mathrm{cm})$} \\
\cline { 2 - 11 } & Itaipu & Mestre & Itaipu & Mestre & Itaipu & Mestre & Itaipu & Mestre & Itaipu & Mestre \\
\hline 0 & $85^{*}$ & 91 & 89 & 95 & 87 & 90 & $2,3 \mathrm{~b}$ & $2,6 \mathrm{a}$ & 5,3 & 6,2 \\
250 & 85 & 92 & 92 & 96 & 85 & 89 & $2,3 \mathrm{~b}$ & $2,8 \mathrm{a}$ & 5,5 & 6,2 \\
500 & 86 & 93 & 92 & 97 & 86 & 88 & $2,4 \mathrm{~b}$ & $2,8 \mathrm{a}$ & 5,3 & 6,3 \\
750 & 91 & 93 & 94 & 96 & 85 & 92 & $2,4 \mathrm{~b}$ & $3,0 \mathrm{a}$ & 5,4 & 6,4 \\
1000 & 90 & 97 & 96 & 98 & 83 & 91 & $2,3 \mathrm{~b}$ & $3,2 \mathrm{a}$ & 5,2 & 6,7 \\
\hline Média & $87 \mathrm{~b}$ & $93 \mathrm{a}$ & $93 \mathrm{~b}$ & $96 \mathrm{a}$ & $85 \mathrm{~b}$ & $90 \mathrm{a}$ & 2,3 & 2,9 & $5,3 \mathrm{~b}$ & $6,4 \mathrm{a}$ \\
\hline C.V. \% & 5,5 & \multicolumn{7}{c}{2,6} & \multicolumn{3}{c}{6,1} & & 6,4 \\
\hline
\end{tabular}

*Médias seguidas por mesma letra, na linha, não diferem entre si pelo teste de Tukey $(\mathrm{p} \leq 0,05)$. ${ }^{* *}$ Dose de biorregulador $\left(\mathrm{mL} \mathrm{ha}^{-1}\right)$, ns: não significativo.

A diferença observada nos testes após o tratamento das sementes com as doses de biorregulador, provavelmente, deu-se pelo fato de que as sementes apresentavam diferentes porcentagens de germinação anteriormente ao tratamento. A cultivar TBIO Itaipu apresentou 89\% de germinação, enquanto que a cultivar TBIO Mestre, 95\%. Semelhantemente, Ramos et al. (2015) [18], observaram que a aplicação do biorregulador Stimulate ${ }^{\circledR}$ em sementes de feijão, a cultivar com qualidade inicial superior apresentou melhor respostas que as demais cultivares utilizadas. Apesar da diferença na qualidade inicial entre as cultivares, é importante destacar que as doses de biorregulador promoveram melhora no desempenho da cultivar TBIO Itaipu, pois proporcionaram incremento de 4 pontos percentuais na germinação, enquanto que para a cultivar TBIO Mestre o incremento foi de apenas um ponto percentual.

Da mesma forma Vendruscolo et al. (2015) [19] observaram através das análises realizadas, comportamento distinto em duas cultivares de algodão, quando submeteram as sementes ao tratamento com doses do biorregulador Stimulate ${ }^{\circledR}$. Comportamento distinto também foram observados por Albrecht et al. (2014) [20], onde as cultivares de ervilha utilizadas responderam de forma diferente ao tratamento das sementes com doses do biorregulador Stimulate ${ }^{\circledR}$, atribuindo tais 
resultados a variabilidade fenotípica dos genótipos em relação à qualidade fisiológica das sementes, e também pela influência direta do biorregulador.

Para a primeira contagem de germinação, germinação e teste de frio é possível observar efeito positivo das doses de biorregulador, na média das cultivares, apresentando incrementos na porcentagem de plântulas normais até a maior dose estudada, sendo observado 93, 97 e 95\% de plântulas normais, o que representou um ganho de 5,8; 4,6 e 5,8 pontos percentuais, em relação à dose zero (Tabela 2).

Tais melhorias na qualidade fisiológica das sementes tratadas com biorregulador, pode ser atribuída ao fato de que estes produtos, possuem em sua formulação compostos, como a giberelina, que estimulam a síntese de enzimas que digerem as reservas armazenadas no endosperma, produzindo açúcares simples, aminoácidos e ácidos nucleicos. Estes compostos são absorvidos e translocados para as regiões de crescimento do embrião, o que estimula o alongamento celular e o rompimento da raiz no tegumento das sementes, proporcionando maior uniformidade na germinação das sementes [21].

Para o comprimento da parte aérea, com a cultivar TBIO Itaipu os resultados referentes as doses de biorregulador não se adequaram a nenhum modelo matemático testado. Já na cultivar TBIO Mestre, os resultados adequaram a um modelo linear crescente, com ganho de $0,6 \mathrm{~cm}$ na maior dose. Em arroz, as doses do biorregulador Stimulate ${ }^{\circledR}$ aplicadas via tratamento de sementes não apresentaram melhorias no comprimento de raiz, na massa de matéria seca da raiz e da parte aérea, no índice de velocidade de emergência e na percentagem de emergência, no entanto, quando utilizado o tratamento na dose de $1000 \mathrm{~mL}$ por $100 \mathrm{~kg}$ de sementes, observou-se aumentos no comprimento da parte aérea, sem prejudicar a germinação das sementes [22]. Ainda de acordo com Elli et al. (2016) [23], a aplicação de doses de biorregulador (0, 3, 6, 9 e $\left.12 \mathrm{~mL} \mathrm{~kg} \mathrm{sementes}{ }^{-1}\right)$ via tratamento de sementes, em diferentes cultivares de arroz, proporcionou incrementos no vigor das sementes de todas as cultivares, aumentando ainda a germinação das sementes de menor qualidade.

Tabela 2. Regressão das doses de biorregulador para a primeira contagem de germinação (PCG), germinação $(G)$, teste de frio $(T F)$ e comprimento da parte aérea $(C P A)$, de sementes de trigo, das cultivares Itaipu e Mestre, em função do tratamento das sementes com doses de biorregulador.

\begin{tabular}{cccccccc}
\hline Variável & Cultivar & Equação & $\mathrm{P}$ & $\mathrm{R}^{2}$ & DME** $^{* *}$ & $\mathrm{Y}_{\mathrm{E}}$ & DDZ \\
\hline \multirow{2}{*}{ PCG } & $\begin{array}{c}\text { TBIO Itaipu } \\
\text { TBIO Mestre }\end{array}$ & $0,0058 \mathrm{x}+87,1^{1}$ & $*$ & 0,92 & 1000 & 93 & 5,8 \\
\hline \multirow{2}{*}{$\mathrm{G}$} & $\begin{array}{c}\text { TBIO Itaipu } \\
\text { TBIO Mestre }\end{array}$ & $0,0046 \mathrm{x}+92^{1}$ & $*$ & 0,92 & 1000 & 97 & 4,6 \\
\hline \multirow{2}{*}{$\mathrm{TF}$} & $\begin{array}{c}\text { TBIO Itaipu } \\
\text { TBIO Mestre }\end{array}$ & $0,0058 \mathrm{x}+88,85^{1}$ & $*$ & 0,88 & 1000 & 95 & 5,8 \\
\hline \multirow{2}{*}{$\mathrm{CPA}$} & $\begin{array}{c}\text { TBIO Itaipu } \\
\text { TBIO Mestre }\end{array}$ & $\begin{array}{c}0,0006 \mathrm{NS}+2,6 \\
\text { N }\end{array}$ & $*$ & 0,94 & 1000 & 3,2 & 0,6 \\
\hline
\end{tabular}

${ }^{1}$ Média das duas cultivares. ${ }^{*}$ Significativo a $5 \%$ de probabilidade de erro. ${ }^{* * D M E: ~ D o s e ~ d e ~ m a ́ x i m a ~}$ eficiência; $\mathrm{Y}_{\mathrm{E}}$ : Valor estimado; DDZ: Diferença da dose zero.

Para as plantas produzidas em campo, oriundas de sementes tratadas com doses de biorregulador (Tabela 3), observa-se que para o peso de sementes por parcela houve interação entre os fatores estudados, onde a cultivar TBIO Mestre foi superior a TBIO Itaipu nas doses de 750 e $1000 \mathrm{~mL}$ $100 \mathrm{~kg}$ sementes $^{-1}$. Já para o peso de mil sementes observa-se diferença, sendo a TBIO Mestre superior a TBIO Itaipu. Sabe-se que o peso das sementes, assim como, a qualidade fisiológica das sementes, são influenciadas pelas características genéticas de cada cultivar e pelas condições em que são produzidas, sofrendo fortemente ação do meio ambiente e dos manejos de cultivos aplicados.

A utilização do Stimulate pode, em função da sua composição, concentração e proporção das substâncias, incrementar o crescimento e desenvolvimento vegetal estimulando a divisão celular, diferenciação e o alongamento das células, podendo também, aumentar a absorção e a utilização de água e dos nutrientes pelas plantas [24]. A cinetina promove a divisão celular, exerce funções reguladoras do desenvolvimento da planta como proliferação de gemas laterais, neoformação de 
órgãos e desenvolvimento do cloroplasto e senescência, sempre em presença de auxinas. Já o ácido indol butílico participa principalmente no crescimento, aumentando a plasticidade da parede celular, o enraizamento, dominância apical, abscisão de flores, estabelecimento dos frutos, etc [25, 26].

Tabela 3. Peso de sementes por parcelas (PSPR) e peso de mil sementes (PMS), de plantas de trigo das cultivares TBIO Itaipu e TBIO Mestre, produzidas em função do tratamento das sementes com doses de biorregulador.

\begin{tabular}{ccccc}
\hline \multirow{2}{*}{ Dose** } & \multicolumn{2}{c}{ PSPR } & \multicolumn{2}{c}{ PMS } \\
\cline { 2 - 5 } & Itaipu & Mestre & Itaipu & Mestre \\
\hline 0 & $695,5 \mathrm{a}^{*}$ & $743,3 \mathrm{a}$ & 31,1 & 33,0 \\
250 & $771,8 \mathrm{a}$ & $736,4 \mathrm{a}$ & 32,6 & 34,2 \\
500 & $785,2 \mathrm{a}$ & $772,3 \mathrm{a}$ & 32,8 & 35,2 \\
750 & $704,8 \mathrm{~b}$ & $816,5 \mathrm{a}$ & 33,2 & 35,0 \\
1000 & $714,9 \mathrm{~b}$ & $799,4 \mathrm{a}$ & 31,9 & 35,2 \\
\hline Média & 734,5 & 773,6 & $32,3 \mathrm{~b}$ & $34,5 \mathrm{a}$ \\
\hline C.V. \% & 5,8 & & & 2,6 \\
\hline
\end{tabular}

*Médias seguidas por mesma letra, na linha, não diferem entre si pelo teste de Tukey $(\mathrm{p} \leq 0,05),{ }^{* *}$ Dose de biorregulador $\left(\mathrm{mL} \mathrm{ha}^{-1}\right)$.

Em relação a qualidade fisiológica das sementes produzidas em campo em função do tratamento de sementes com doses de biorregulador (Tabela 4), observa-se que apenas a variável emergência não apresentou efeitos significativos entre os fatores estudados. Para as demais variáveis pode ser observado efeito principal para o fator cultivar, onde a cultivar TBIO Mestre foi superior a cultivar TBIO Itaipu. Estas diferenças observadas entre as cultivares, podem ser em função das características genéticas de cada cultivar, que podem proporcionar melhor adaptação de uma cultivar em determinado local de cultivo.

Alterações na concentração hormonal nos tecidos das plantas podem mediar toda uma gama de processos de desenvolvimento das plantas, muitos dos quais envolvem interações com os fatores ambientais [27]. Desta forma, acredita-se que as características da cultivar TBIO Mestre, juntamente com as condições de ambiente onde as plantas foram cultivadas, possam ter interagido com as doses de biorregulador aplicadas no tratamento das sementes, resultando em melhor desempenho, em relação a cultivar TBIO Itaipu.

$\mathrm{Na}$ Tabela 5, são apresentadas as equações de regressão das doses de biorregulador realizada para as variáveis peso de sementes por parcela, peso de mil sementes, primeira contagem de germinação, germinação e teste de frio. Para o peso de sementes por parcelas, as doses de biorregulador responderam de forma diferente para as duas cultivares de trigo, sendo que para a cultivar TBIO Itaipu os resultados adequaram-se a um modelo quadrático positivo, com ponto de máxima eficiência na dose de $412 \mathrm{~mL} \mathrm{ha}^{-1}$, onde obteve $758,7 \mathrm{~g}$ por parcela. Já para a cultivar TBIO Mestre os resultados adequaram-se a modelo linear crescente, obtendo na maior dose 812,1 g por parcela. Esses resultados revelam um ganho de 50,9 e $77 \mathrm{~g}$ em relação a dose zero, respectivamente, para cada cultivar nas respectivas doses de máxima eficiência técnica. 
Tabela 4. Porcentagem de plântulas normais obtidas nos testes de primeira contagem de germinação $(P C G)$, germinação $(G)$, teste de frio $(T F)$, envelhecimento acelerado $(E A)$, emergência em campo $(E C)$, comprimento da parte aérea (CPA) e comprimento de raiz (CR) de sementes de trigo das cultivares TBIO Itaipu e TBIO Mestre, produzidas em função do tratamento das sementes com doses de biorregulador.

\begin{tabular}{|c|c|c|c|c|c|c|}
\hline \multirow{2}{*}{$\operatorname{Dose}^{* *}$} & \multicolumn{2}{|c|}{ PCG $(\%)$} & \multicolumn{2}{|c|}{$\mathrm{G}(\%)$} & \multicolumn{2}{|c|}{$\mathrm{TF}(\%)$} \\
\hline & Itaipu & Mestre & Itaipu & Mestre & Itaipu & Mestre \\
\hline 0 & $84 *$ & 90 & 88 & 91 & 86 & 91 \\
\hline 250 & 85 & 92 & 90 & 96 & 91 & 92 \\
\hline 500 & 85 & 95 & 91 & 99 & 92 & 97 \\
\hline 750 & 90 & 94 & 94 & 96 & 95 & 96 \\
\hline 1000 & 82 & 89 & 85 & 92 & 85 & 91 \\
\hline Média & $85 \mathrm{~b}$ & $92 \mathrm{a}$ & $90 \mathrm{~b}$ & $94 \mathrm{a}$ & $90 \mathrm{~b}$ & $93 \mathrm{a}$ \\
\hline C.V. \% & & & & & & \\
\hline \multirow{2}{*}{ Dose } & \multicolumn{2}{|c|}{ EA (\%) } & \multicolumn{2}{|c|}{$\mathrm{CPA}(\mathrm{cm})$} & \multicolumn{2}{|c|}{$\mathrm{CR}(\mathrm{cm})$} \\
\hline & Itaipu & Mestre & Itaipu & Mestre & Itaipu & Mestre \\
\hline 0 & 86 & 89 & 3,1 & 3,1 & 5,2 & 5,6 \\
\hline 250 & 88 & 89 & 3,0 & 3,2 & 5,8 & 6,3 \\
\hline 500 & 86 & 93 & 2,9 & 3,4 & 5,5 & 6,4 \\
\hline 750 & 83 & 97 & 3,2 & 3,2 & 5,4 & 6,2 \\
\hline 1000 & 85 & 87 & 2,9 & 3,2 & 5,5 & 6,4 \\
\hline Média & $86 \mathrm{~b}$ & $91 \mathrm{a}$ & $3 b$ & $3,2 \mathrm{a}$ & $5,5 \mathrm{~b}$ & $6,2 \mathrm{a}$ \\
\hline C.V. \% & \multicolumn{2}{|c|}{5,9} & \multicolumn{2}{|c|}{6,3} & \multicolumn{2}{|c|}{7,1} \\
\hline
\end{tabular}

*Médias seguidas por mesma letra, na linha, não diferem entre si pelo teste de Tukey $(\mathrm{p} \leq 0,05) .{ }^{* *}$ Dose de biorregulador $\left(\mathrm{mL} \mathrm{ha}^{-1}\right)$, ns: não significativo.

Tabela 5. Regressão das doses de biorregulador para o peso de sementes por parcela (PSPR), peso de mil sementes $(P M S)$, primeira contagem de germinação $(P C G)$, germinação $(G)$ e teste de frio $(T F)$, de sementes de trigo das cultivares Itaipu e Mestre, produzidas em função do tratamento das sementes com biorregulador.

\begin{tabular}{cccccccc}
\hline Variável & Cultivar & Equação & $\mathrm{P}$ & $\mathrm{R}^{2}$ & $\mathrm{DME}$ & $\mathrm{Y}^{\mathrm{E}}$ & $\mathrm{DDZ}$ \\
\hline \multirow{2}{*}{ PSPR } & TBIO Itaipu & $0,0003 \mathrm{x}^{2}+0,2472 \mathrm{x}+707,78$ & $*$ & 0,55 & 412 & 758,7 & 50,9 \\
& TBIO Mestre & $0,077 \mathrm{x}+735,09$ & $*$ & 0,77 & 1000 & 812,1 & 77 \\
\hline \multirow{2}{*}{ PMS } & $\begin{array}{c}\text { TBIO Itaipu } \\
\text { TBIO Mestre }\end{array}$ & $-5 \mathrm{e}-06 \mathrm{x}^{2}+0,00491 \mathrm{x}+32,491^{1}$ & $*$ & 0,99 & 491 & 33,7 & 1,2 \\
\hline \multirow{2}{*}{ PCG } & $\begin{array}{c}\text { TBIO Itaipu } \\
\text { TBIO Mestre }\end{array}$ & $-2 \mathrm{e}-05 \mathrm{x}^{2}+0,017 \mathrm{x}+86,243^{1}$ & $*$ & 0,59 & 425 & 90 & 3,6 \\
\hline \multirow{2}{*}{$\mathrm{G}$} & $\begin{array}{c}\text { TBIO Itaipu } \\
\text { TBIO Mestre }\end{array}$ & $-2 \mathrm{e}-05 \mathrm{x}^{2}+0,0238 \mathrm{x}+89,05^{1}$ & $*$ & 0,91 & 595 & 96 & 7 \\
\hline \multirow{2}{*}{ TF } & $\begin{array}{c}\text { TBIO Itaipu } \\
\text { TBIO Mestre }\end{array}$ & $-3 \mathrm{e}-05 \mathrm{x}^{2}+0,0275 \mathrm{x}+87,514^{1}$ & $*$ & 0,83 & 458 & 94 & 6,3 \\
\hline
\end{tabular}

${ }^{1}$ Média das duas cultivares. ${ }^{*}$ Significativo a $5 \%$ de probabilidade de erro. **DME: Dose de máxima eficiência; $\mathrm{Y}_{\mathrm{E}}$ : Valor estimado; DDZ: Diferença da dose zero.

O biorregulador Stimulate ${ }^{\circledR}$ aplicado via tratamento de sementes de trigo, no intervalo das doses entre 3,5 e 5,0 mL kg sementes ${ }^{-1}$, proporcionou aumentos significativos na altura e massa de matéria seca da parte aérea, ainda doses crescentes proporcionaram aumentos lineares no crescimento radicular vertical e total e na velocidade de crescimento radicular vertical, no entanto a porcentagem 
de plântulas normais, o vigor de plântulas e a produção de grãos por planta não foram influenciados [28]. O tratamento de sementes de arroz com doses do biorregulador Stimulate ${ }^{\circledR}(0 ; 250 ; 500 ; 750$ e $1000 \mathrm{~mL} 100 \mathrm{~kg}$ sementes $^{-1}$ ), não promoveu melhoras na germinação, nem aumentou o comprimento de raiz, a massa de matéria seca de raiz e parte aérea, o índice de velocidade e a percentagem de emergência, no entanto as doses crescentes aumentaram o comprimento da parte aérea [22].

No que se refere ao peso de mil sementes, os resultados referentes as doses de biorregulador, para as médias das cultivares, adequaram-se a um modelo polinomial quadrático positivo, com ponto de máxima eficiência nas doses de $491 \mathrm{~mL} \mathrm{ha}^{-1}$, resultado em um ganho de 1,2 gramas em relação a dose zero, respectivamente. Para a primeira contagem de germinação, germinação e teste de frio, as regressões para as doses de biorregulador adequaram a um modelo polinomial quadrático positivo, com pontos de máxima nas doses de 425,595 e $458 \mathrm{~mL} \mathrm{ha}^{-1}$, obtendo nestas doses um percentual de plântulas normais de 90, 96 e 94 \%, com uma diferença de 3,6; 7 e 6,3 pontos percentuais a mais, em relação a doze zero, respectivamente.

Alguns autores, como Vieira e Castro (2002) [29] atribuem as melhorias observadas pela aplicação do biorregulador Stimulate ${ }^{\circledR}$, ao fato, de que estes atuam de maneira eficaz na germinação de sementes, no vigor inicial das plântulas, no crescimento e desenvolvimento radicular e foliar, bem como na produção de compostos orgânicos, incrementando assim, o crescimento e desenvolvimento vegetal, estimulando a divisão celular, podendo ainda, aumentar a absorção de água e de nutrientes pelas plantas, o que pode resultar em aumentos de produção, assim como na obtenção de sementes com qualidade superior.

\subsection{Experimento 2- Pulverização foliar}

Em relação aos resultados obtidos com a aplicação foliar do biorregulador, observou-se que para o peso de sementes por parcelas, primeira contagem de germinação, germinação e emergência em campo não houve significância estatística para os fatores estudados (dados não apresentados). Para o peso de mil sementes, observa-se que a cultivar TBIO Mestre foi superior a cultivar TBIO Itaipu, mostrando maior resposta desta cultivar a aplicação das doses de biorregulador, pois segundo seus descritores morfológicos, ambas cultivares apresentam peso de mil sementes idênticos (Tabela 6).

Em relação a qualidade fisiológica das sementes produzidas em campo em função da aplicação de doses de biorregulador via foliar (Tabela 6), observa-se que para as variáveis primeira contagem de germinação, germinação e emergência em campo não houveram efeitos significativos entre os fatores estudados. Já para as demais variáveis observa-se efeito principal para o fator cultivar, onde a cultivar TBIO Mestre foi superior a cultivar TBIO Itaipu em todas as variáveis analisadas.

Tabela 6. Peso de mil sementes (PMS), teste de frio (TF), envelhecimento acelerado (EA), comprimento da parte aérea (CPA) e comprimento de raiz (CR) de sementes de trigo das cultivares TBIO Itaipu e TBIO Mestre, produzidas em função da aplicação foliar de doses de biorregulador.

\begin{tabular}{ccccccccccc}
\hline \multirow{2}{*}{ Dose*** } & \multicolumn{3}{c}{ PMS (g) } & \multicolumn{2}{c}{ TF $(\%)$} & \multicolumn{2}{c}{ EA (\%) } & \multicolumn{2}{c}{ CPA $(\mathrm{cm})$} & \multicolumn{2}{c}{ CR $(\mathrm{cm})$} \\
\cline { 2 - 11 } & Itaipu & Mestre & Itaipu & Mestre & Itaipu & Mestre & Itaipu & Mestre & Itaipu & Mestre \\
\hline 0,0 & 33,3 & 34,3 & 87 & 90 & 84 & 94 & 3,0 & 3,2 & 5,7 & 6,2 \\
187,5 & 35,4 & 35,8 & 94 & 94 & 87 & 94 & 3,0 & 3,4 & 5,5 & 7,1 \\
375,0 & 35,2 & 36,1 & 92 & 97 & 87 & 94 & 3,0 & 3,1 & 5,5 & 6,4 \\
562,5 & 35,5 & 35,9 & 90 & 94 & 85 & 90 & 2,9 & 3,1 & 5,4 & 6,4 \\
750,0 & 34,6 & 36,7 & 90 & 94 & 83 & 95 & 3,0 & 3,2 & 5,7 & 6,1 \\
\hline Média & $34,8 \mathrm{~b}$ & $35,8 \mathrm{a}$ & $90 \mathrm{~b}$ & $94 \mathrm{a}$ & $85 \mathrm{~b}$ & $93 \mathrm{a}$ & $3,0 \mathrm{~b}$ & $3,2 \mathrm{a}$ & $5,6 \mathrm{~b}$ & $6,5 \mathrm{a}$ \\
\hline C.V. \% & \multicolumn{3}{c}{3,0} & \multicolumn{3}{c}{3,8} & \multicolumn{3}{c}{6,7} & \multicolumn{2}{c}{9,6} \\
\hline
\end{tabular}

*Médias seguidas por mesma letra, na linha, não diferem entre si pelo teste de Tukey $(\mathrm{p} \leq 0,05)$. ${ }^{* *}$ Dose de biorregulador $\left(\mathrm{mL} \mathrm{ha}^{-1}\right)$, ns: não significativo. 
Alguns autores relatam que a produtividade, assim como a qualidade fisiológica das sementes é mais influenciada pelas condições ambientais prevalecentes durante a fase de maturação e colheita das sementes, do que pelas características da própria cultivar [30, 31, 32]. Em contrapartida Paschalii e Ellis (1978) [33] e Krzyzanowski et al. (1993) [34], descrevem que o fator determinante e fundamental da qualidade fisiológica das sementes é intrínseco e dependente do controle genético dessa característica pela cultivar, o que pode justificar as diferenças de respostas das cultivares, a aplicação foliar do biorregulador. Apesar de serem observadas diferenças significativas entre as cultivares, cabe ainda, salientar o efeito das doses do biorregulador (Tabela 6), a qual demonstra um comportamento semelhante entre as cultivares, apenas sendo mais acentuado em uma das cultivares.

As regressões das doses de biorregulador para as variáveis peso de mil sementes, primeira contagem de germinação, germinação e teste de frio (Tabela 7) se adequaram a um modelo quadrático positivo para todas as variáveis, apresentando pontos de máxima nas doses de 481, 442, 440 e $378 \mathrm{~mL} \mathrm{ha}^{-1}$, respectivamente, para os parâmetros citados, com acréscimo de 1,8 g no peso de mil sementes e de 5,9; 5,8 e 4,3 pontos percentuais para a primeira contagem de germinação, germinação e teste de frio, em relação a dose zero, respectivamente.

Tabela 7. Regressão das doses de biorregulador para o peso de mil sementes (PMS), primeira contagem de germinação (PCG), germinação $(G)$ e teste de frio $(T F)$, de sementes de trigo das cultivares Itaipu e Mestre, produzidas em função da aplicação foliar de biorregulador.

\begin{tabular}{cccccccc}
\hline Variável & Cultivar & Equação & $\mathrm{P}$ & $\mathrm{R}^{2}$ & $\mathrm{DME}^{* *}$ & $\mathrm{Y}_{\mathrm{E}}$ & $\mathrm{DDZ}$ \\
\hline \multirow{2}{*}{ PMS } & $\begin{array}{c}\text { TBIO Itaipu } \\
\text { TBIO Mestre }\end{array}$ & $-8 \mathrm{E}-06 \mathrm{x}^{2}+0,0077 \mathrm{x}+33,991^{1}$ & $*$ & 0,88 & 481 & 35,8 & 1,8 \\
\hline \multirow{2}{*}{ PCG } & $\begin{array}{c}\text { TBIO Itaipu } \\
\text { TBIO Mestre }\end{array}$ & $-0 \mathrm{E}-05 \mathrm{x}^{2}+0,0265 \mathrm{x}+85,079^{1}$ & $*$ & 0,89 & 442 & 91 & 5,9 \\
\hline \multirow{2}{*}{$\mathrm{G}$} & $\begin{array}{c}\text { TBIO Itaipu } \\
\text { TBIO Mestre }\end{array}$ & $-3 \mathrm{E}-05 \mathrm{x}^{2}+0,0264 \mathrm{x}+87,671^{1}$ & $*$ & 0,98 & 440 & 93 & 5,8 \\
\hline \multirow{2}{*}{ TF } & $\begin{array}{c}\text { TBIO Itaipu } \\
\text { TBIO Mestre }\end{array}$ & $-3 \mathrm{E}-05 \mathrm{x}^{2}+0,227 \mathrm{x}+89,157^{1}$ & $*$ & 0,76 & 378 & 94 & 4,3 \\
\hline
\end{tabular}

${ }^{1}$ Média das duas cultivares. *Significativo a 5\% de probabilidade de erro. **DME: Dose de máxima eficiência; $\mathrm{Y}_{\mathrm{E}}$ : Valor estimado; DDZ: Diferença da dose zero.

A aplicação do biorregulador Stimulate ${ }^{\circledR}$ no tratamento de sementes de milho $(500,1000$ e 1500 $\mathrm{mL} 100 \mathrm{~kg}$ sementes $\left.^{-1}\right)$, através de pulverização na linha de semeadura $\left(500,1000\right.$ e $\left.1500 \mathrm{~mL} \mathrm{ha}^{-1}\right)$ e em pulverização foliar $\left(250,500\right.$ e $\left.750 \mathrm{~mL} \mathrm{ha}^{-1}\right)$, não promoveram aumento no peso de mil sementes, no entanto, promoveu aumentos no número de grãos em cada fileira da espiga, o que resultou em maior produtividade, sendo mais efetivo quando aplicado via tratamento de sementes [10]. Já o uso do biorregulador Stimulate ${ }^{\circledR}$ em feijoeiro, aplicado via foliar na fase vegetativa não interferiu no crescimento da parte aérea, nem na produtividade [35].

Já em relação a qualidade fisiológica de sementes, o aumento das doses de biorregulador Stimulate ${ }^{\circledR}$ promoveu incremento no vigor das sementes de Brachiaria decumbens, promovendo aumentos lineares na parte aérea das plântulas, maior índice de germinação e maior comprimento

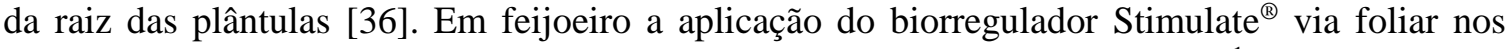
estádios de desenvolvimento V4 e V5, nas doses de $0 ; 0,5 ; 1 ; 1,5$ e $2 \mathrm{~L} \mathrm{ha}^{-1}$, não afetaram a porcentagem de germinação, a primeira contagem de germinação, o envelhecimento acelerado, $o$ teste de frio, a condutividade elétrica, o comprimento de plântulas, a emergência em campo, o índice de velocidade de emergência, a altura de plantas e a massa verde e seca de plântulas, porém foi observado influência significativa na porcentagem de plântulas vigorosas [37].

O manejo da cultura do trigo com o biorregulador Stimulate ${ }^{\circledR}$ aplicado no perfilhamento das plantas, em duas safras consecutivas, não incrementou a qualidade fisiológica das sementes, porém favoreceu o aumento do teor de proteínas das sementes [11]. De forma semelhante Navarini (2010) [38], não observou diferenças entre os tratamentos com os biorreguladores Stimulate ${ }^{\circledR}$ e Booster ${ }^{\circledR}$, para o potencial de germinação das sementes de trigo produzidas. 
Em outro trabalho realizado com trigo, foi observado superioridade dos tratamentos com o biorregulador Stimulate ${ }^{\circledR}$ em relação a testemunha, com aumentos significativos de $10 \%$ para o peso de mil sementes [12], sendo o peso de mil sementes um importante indicativo da qualidade de sementes, pois Hessel et al. (2012) [39], destacaram que a superioridade do peso de mil sementes pode estar relacionada com o maior potencial fisiológico das sementes. Da mesma forma, Barbosa et al. (2010) [40] observaram que sementes com maior peso de mil, obtiveram maior expressão do vigor durante $\mathrm{o}$ armazenamento das sementes.

\section{CONCLUSÃO}

A utilização de Stimulate ${ }^{\circledR}$ exerce efeito diferenciado nas cultivares de trigo, influenciado pela genética.

O biorregulador Stimulate ${ }^{\circledR}$ aplicado via foliar nos estádios V3 e R1, nas doses próximas de 450 $\mathrm{mL} \mathrm{ha} \mathrm{a}^{-1}$, ou via tratamento de sementes até $1000 \mathrm{~mL} 100 \mathrm{~kg}$ sementes ${ }^{-1}$, podem promover acréscimos no rendimento de sementes, no peso de mil sementes e na qualidade fisiológica das sementes produzidas, dependendo da cultivar de trigo.

\section{REFERÊNCIAS BIBLIOGRÁFICAS}

1. Scheuer PM, Francisco A, Miranda MZ, Limberger VM. Trigo: características e utilização na panificação. Rev Bras Prod Agroind. 2011;13(2):211-222. doi:10.15871/1517-8595/rbpa.v13n2p211-222

2. Tavares LC, Rufino CA, Brunes AP, Friedrich FF, Barros ACSA, Villela FA. Physiological performance of wheat seeds coated with micronutrients. J Seed Sci. 2013;35(1):28-34, doi:10.1590/S231715372013000100004.

3. Peske ST, Barros ACSA, Schuch LOB. Produção de sementes. IN.: Peske ST, Villela FA, Meneghello GE. (Orgs.) Sementes: Fundamentos Científicos e tecnológicos. 3. ed. Pelotas: Editora Universitária/UFPel, 2012. p. 13-104.

4. Baudet L, Peres WB. Recobrimento de sementes. Seed News. 2004;4(1):20-23.

5. Sanches FR. Aplicação de biorreguladores vegetais: aspectos fisiológicos e aplicações práticas na citricultura mundial. Jaboticabal: Funep, 2000. 160p.

6. Taiz L, Zeiger E, Moller IM, Murphy A. Fisiologia e Desenvolvimento vegetal. Porto Alegre: Artmed, 6 ed, 2017, 858p.

7. Vieira EL, Castro PRC. Ação de bioestimulante na germinação de sementes, vigor das plântulas, crescimento radicular e produtividade de soja. Rev Bras Sementes. 2001;23(2):222-228, doi:10.17801/0101-3122/rbs.v23n2p222-228.

8. Silva TT de A, Von Pinho EVR, Cardoso DL, Ferreira CA, Alvim PO, Costa AAF. Qualidade fisiológica de sementes de milho na presença de bioestimulantes. Ciênc Agrotec. 2008;32(3):840-846, doi:10.1590/S1413-70542008000300021

9. Vasconcelos ACF de. Uso de bioestimulantes nas culturas de milho e soja. [tese]. Piracicaba (SP): Escola Superior de Agricultura Luiz de Queiroz, 2006. 111p.

10. Dourado Neto D, Dario GJA, Barbieri APP, Martin TN. Ação de bioestimulante no desempenho agronômico de milho e feijão. Biosc J. 2014;30(1):371-379.

11. Piccinin GG, Braccini AL, Dan LGM, Scapim CA, Mariucci GEG, Suzukawa AK. Inoculação de sementes de trigo com Azospirillum brasilense associada a aplicação de biorregulador. Informativo ABRATES. 2015;25(1):30-38.

12. Mendes MC, Gabriel A, Vidal LHI, Faria MV, Possato Junior O, Camargo Junior OA. Biorregulador aplicado em diferentes estádios fenológicos na cultura do trigo. Rev Agro@ mbiente. 2015;9(4):476-480, doi:10.18227/1982-8470ragro.v9i4.2620

13. Nunes JC. Tratamento de semente - qualidade e fatores que podem afetar a sua performance em laboratório. Syngenta Proteção de Cultivos Ltda. 2005. 16p.

14. Brasil. Ministério da Agricultura, Pecuária e Abastecimento. Regras para análise de sementes. Brasília: Mapa/ACS, 2009. 399p.

15. Cícero SM, Vieira, RD. Teste de frio. In: Vieira RD, Carvalho NM. (Ed.) Testes de vigor em sementes. Jaboticabal: FUNEP, 1994. p.151-164.

16. Marcos Filho J. Fisiologia de sementes de plantas cultivadas. ed. 5. Londrina: Abrates. 2015, 659p.

17. Nakagawa J. Testes de vigor baseados no desempenho das plântulas. In: Krzyzanowski FC, Vieira RD, França-Neto JB. Vigor de sementes: conceitos e testes. Londrina: ABRATES, 1999. Cap. 2, p. 9-13.

18. Ramos AR, Binotti, FFS, Silva TR, Silva UR. Bioestimulante no condicionamento fisiológico e tratamento de sementes de feijão. Rev Bioc. 2015 21(1):76-88. 
19. Vendruscolo EP, Souza HB, Arruda LA, Lima SF, Alvarez RCF. Biorregulador na germinação e desenvolvimento inicial de algodoeiro. Rev Ciênc Agroamb. 2015;13(2):32-40.

20. Albrecht LP, Bazo GL, Demeneck-Vieira PV, Albrecht AJP, Braccini AL, Krenchinski FH, Gasparotto AC. Desempenho fisiológico das sementes de ervilha tratadas com biorregulador. Comunicata Scientiae. 2014;5(4):464-470, doi:10.14295/cs.v5i4.350

21. Hopkins WG. The role of hormones in plant development. In: Introduction to plant physiology. 2. ed. New York: John Wiley \& Sons, 1999.

22. Rodrigues LA, Batista MS, Alvares RCF, Lima SF, Alves CZ. Avaliação fisiológica de sementes de arroz submetidas a doses de bioestimulante. Nucleus. 2015;12(1):207-2014, doi:10.3738/1982.2278.1376

23. Elli EF, Monteiro GC, Kulczinski SM, Carone BO, Souza VQ. Potencial fisiológico de sementes de arroz tratadas com biorregulador vegetal. Rev Ciênc Agron. 2016;47(2):366-373.

24. Stoller do Brasil. Stimulate Mo em hortaliças: informativo técnico. Cosmópolis: Stoller do Brasil, Divisão Arbore, 1998, 1p.

25. Azcon-Bieto J, Talón, M. Fundamentos de fisiología vegetal. McGraw-Hill, Madrid, España. 2003. 522p

26. Castro PRC, Serciloto CM, Pereira MA, Rodrigues JLM, Rossi G. Agroquímicos de controle hormonal, fosfitos e potencial de aplicação dos aminoácidos na agricultura tropical. Piracicaba: DIBD-ESALQ, (Série Produtor Rural). 2009, 83p.

27. Crozier A, Kamiya Y, Bishop G, Yokota T. Biosynthesis of hormones and elicitor molecules. In.: Buchanan BB, Grissen W, Jones RL. (eds.). Biochemistry and Molecular Biology of Plants. American Society of Plant physiologists, Rockiville, Maryland, 2000, p.850-894.

28. Cato SC. Ação de biostimulante nas culturas do amendoinzeiro, sorgo e trigo e interações hormonais entre auxinas, citocininas e giberelinas, 2006. 74p. Tese (Doutorado) - Escola Superior de Agricultura "Luiz de Queiros" - Universidade de São Paulo, Piracicaba. 2006.

29. Vieira EL, Castro PRC. Ação de stimulate no desenvolvimento inicial de plantas de algodoeiro (Gossypium hirsutum L.). Piracicaba: USP. Departamento de Ciências Biológicas, 2002. 3 p.

30. Tekrony DM, Egli DB, Balles J, Tomes LJ, Stuckey RE. Effect of date of harvest maturity on soybean seed quality and Phomopsis sp. seed infection. Crop Sci. 1984;24(1):189-93. doi:10.2135/cropsci1984.0011183X002400010045x

31. Vieira RD, Carvalho NM, Sader R. Testes de vigor e suas possibilidades de uso. In: Vieira RD, Carvalho NM. (eds.) Testes de vigor em sementes. Jaboticabal: FUNEP, 1994. p.31- 47.

32. Aguero JAP, Vieira RD, Bittencourt SRM. Avaliação da qualidade fisiológica de sementes de cultivares de soja. Rev Bras Sementes. 1997;19(2):254-259, doi:10.1590/S0100-204X1999000600018

33. Paschalii EH, Ellis MA. Variation in seed quality characteristics on tropically grown soybeans. Crop Sci. 1978;18(3):837-40. doi:10.2135/cropsci1978.0011183X001800050039x

34. Krzyzanowski FC, Gilioli JL, Miranda LC. Produção de sementes nos cerrados. In: Arantes NE, Souza PIM. (eds.) Cultura da soja nos cerrados. Piracicaba: POTAFOS, 1993. p. 465-513.

35. Almeida AQ, Soratto RP, Broetto F, Cataneo AC. Nodulação, aspectos bioquímicos, crescimento e produtividade do feijoeiro em função da aplicação de bioestimulante. Semina: Ciênc Agrárias. 2014;35(1):77-88. doi:10.5433/1679-0359.2014v35n1p77

36. Brennecke K, Ferraz FM, Simões TR. Germinação de sementes de Brachiaria decumbens sob diferentes concentrações de biorregulador. Rev Acad Ciênc Animal. 2015;13:145151, doi:10.7213/academica.13.FC.AO15

37. Abrantes FL. Efeito de bioestimulante sobre a produtividade e qualidade fisiológica de dois cultivares de feijão cultivados no inverno. [dissertação]. Ilha Solteira (SP): Programa de Pós-Graduação em Sistemas de Produção. Faculdade de Engenharia - UNESP - Campus de Ilha Solteira. 2008. 68p.

38. Navarini LL. Manejo do solo e utilização de bioestimulantes na cultura do trigo (Triticum aestivum L.). [dissertação]. Passo Fundo (RS): Universidade de Passo Fundo. 2010. 96p.

39. Hessel CLE, Villela FA, Aumonde TZ, Pedó T. Mesa densimétrica e qualidade fisiológica de sementes de brachiária. Informativo ABRATES. 2012, 22(3):73-76.

40. Barbosa CZR, Smiderle OJ, Alves JMAA, Vilarinho AA, Sediyama, T. Qualidade de sementes de soja BRS Tracajá, colhidas em Roraima em função do tamanho no armazenamento. Rev Ciênc Agron. 2010;41(1):73-80. 